\title{
GLOBAL ICRF SYSTEM DESIGNS FOR ITER AND TPX
}

\author{
R. H. Goulding, D. J. Hoffman, and P. M. Ryan \\ Oak Ridge National Laboratory, Oak Ridge, TN, 37831-8071 \\ F. Durodié \\ Laboratoire de Physique des Plasmas - Association "EURATOM-Etat Belge"- \\ Ecole Royale Militaire - B1040 Brussels, Belgium
}

\begin{abstract}
The design of feed networks for ICRF antenna arrays on ITER and TPX are discussed. Features which are present in one or both of the designs include distribution of power to several straps from a single generator, the capability to vary phases of the currents on antenna elements rapidly without the need to rematch, and passive elements which present a nearly constant load to the generators during ELM induced loading transients of a factor of 10 or more. The FDAC (Feedline/Decoupler/Antenna Calculator) network modeling code is described, which allows convenient modeling of the electrical performance of nearly arbitrary ICRF feed networks.
\end{abstract}

\section{INTRODUCTION}

The present designs of ICRF launchers for both the ITER and TPX feature wide bandwidth arrays with strong coupling between antenna elements. It is required that phasing between elements be variable over millisecond time scales to switch between heating and current drive modes. The new ITER in-port design calls for a power limit of $2 \mathrm{MW} / \mathrm{strap}$ with plasma loading as low as $3 \Omega / \mathrm{m}$. Finally, it is highly desirable to have systems able to deliver a large fraction of the available power in the presence of ELMs, which can cause the resistive loading to increase by a factor of 10 within $1 \mathrm{~ms}$. Specific designs of feed networks for ITER and TPX which meet these goals are discussed in this paper.

\section{MODELING THE ELECTRICAL PERFORMANCE OF ICRF ANTENNA ARRAYS AND FEED SYSTEMS}

FDAC is a network modeling code specifically written to model feed networks for multiple strap ICRF antenna arrays and the arrays themselves. Models are constructed from elements including lossy coupled and uncoupled transmission lines, lumped elements, and components such as hybrid power splitters, connected to form a network. The code calculates impedance or scattering matrices for arbitrary locations in the network, currents and voltages at all locations, and optimum settings for matching and decoupling elements. Parameters including frequency, antenna section lengths, characteristic impedances, phase velocities, resistive and reactive loading, transmission line lengths, tuning element settings, and boundary conditions such as magnitudes and phases of voltages at the generators or currents at the antenna grounds can be scanned over an arbitrary range of values, either

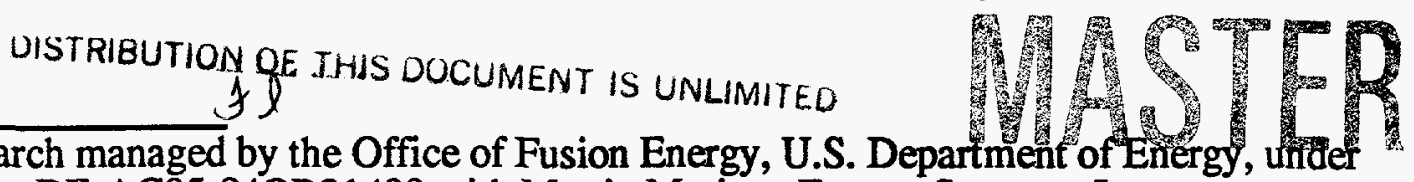
*Research managed by the Office of Fusion Energy, U.S. Department of Energy, uñted contract DE-AC05-84OR21400 with Martin Marietta Energy Systems, Inc. 


\section{DISCLAIMER}

This report was prepared as an account of work sponsored by an agency of the United States Government. Neither the United States Government nor any agency thereof, nor any of their employees, makes any warranty, express or implied, or assumes any legal liability or responsibility for the accuracy, completeness, or usefulness of any information, apparatus, product, or process disclosed, or represents that its use would not infringe privately owned rights. Reference herein to any specific commercial product, process, or service by trade name, trademark, manufacturer, or otherwise does not necessarily constitute or imply its endorsement, recommendation, or favoring by the United States Government or any agency thereof. The views and opinions of authors expressed herein do not necessarily state or reflect those of the United States Government or any agency thereof. 


\section{DISCLAIMER}

Portions of this document may be illegible in electronic image products. Images are produced from the best available original document. 
TABLE 1. TTER/TPX Design Parameters

\begin{tabular}{lcc}
\hline & ITER & TPX \\
\hline Number of straps /elements & $8 / 16$ & $6 / 12$ \\
Number of generators & 8 & 3 \\
Power per strap & $2 \mathrm{MW}$ & $1 \mathrm{MW}$ \\
Frequency range (extended) & $40-70(90) \mathrm{MHz}$ & $40-80 \mathrm{MHz}$ \\
Relative current phasing & arbitrary & arbitrary \\
Peak operating if E field & $1.8 \mathrm{MV} / \mathrm{m}$ & - \\
Peak operating voltage & -- & $30 \mathrm{kV}$ \\
\hline
\end{tabular}

${ }^{\star}$ Between generators

individually or in combination. FDAC has been used for preliminary design studies of feed networks for ICRF antenna arrays for ITER and TPX, and is currently being used to optimize these designs.

\section{ITER ICRF SYSTEM DESIGN}

The present ITER ICRF launcher design is an in-port array consisting of 8 center grounded straps arranged in 2 rows of 4 straps each, for a total of 16 separately matched and fed elements. Design requirements for the launcher and feed networks for both the ITER and TPX arrays are summarized in Table $1 .{ }^{1,2}$

In each port, there are two four-strap modules. A schematic showing the feed circuit for one of the two modules is shown in Fig. 1. In this design each of the 8 elements is matched individually using a mechanical single grounded-stub (S) / line stretcher (T) combination. A generator $(G)$ feeds each hybrid power splitter $(\mathrm{H})$, which in turn feeds each pair of poloidally separated antenna elements (A). There is a decoupler connected across each pair of toroidally spaced elements consisting of a transmission line connected across the corresponding feed lines, with a grounded stub (D) located half way in between the feedlines.

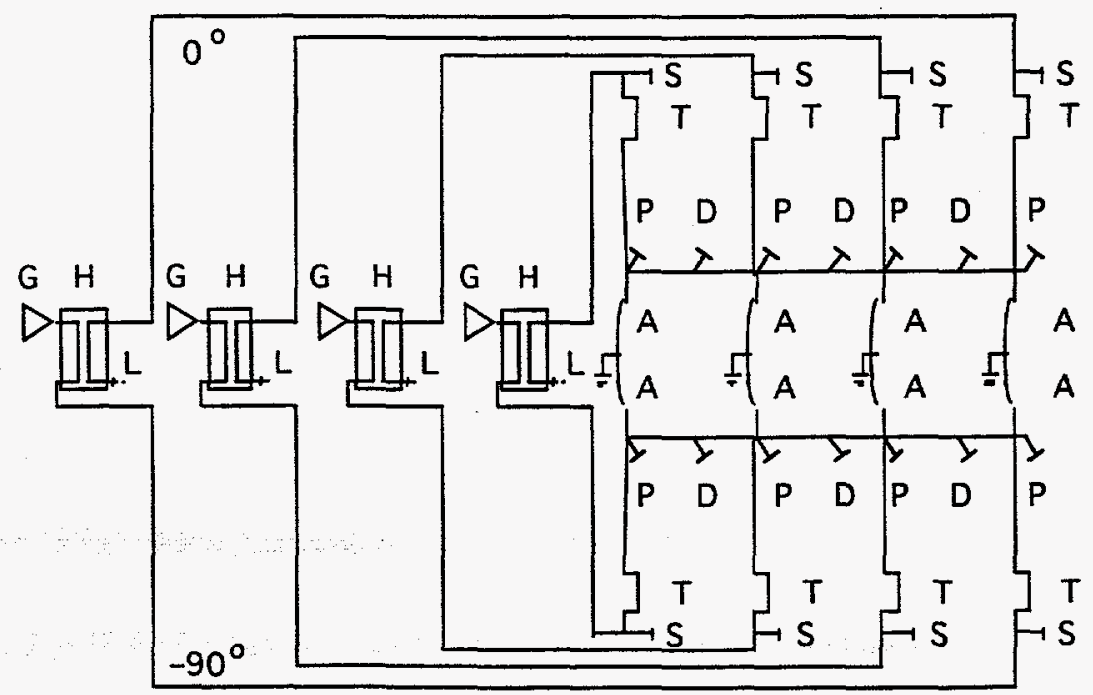

Fig. 1 ITER feed circuit schematic 
The stub adjusts the power flow between the feedlines so that the mutual admittance between the associated antennas is canceled out exactly. Pre-matching stubs (P) are located at the tees connecting the feedlines to the decouplers. These reduce voltages and raise efficiencies in the feedlines over much of the frequency range. Assuming a $30 \Omega$ characteristic impedance for all feedlines, the maximum voltage occurring anywhere in the system is $44 \mathrm{kV}$ for an antenna loading of $R^{\prime}=3 \Omega / \mathrm{m}$.

A very interesting feature of the ITER feed circuit design is its ability to present a nearly constant load to the generators despite order of magnitude increases in resistive loading caused by ELMs, without the need for fast tuning components. The forward voltage waves traveling from the two ports of each hybrid splitter differ in phase by $\pi / 2$, as indicated in figure 1 . Assuming that an ELM produces equal changes in loading on each poloidally separated strap, then the reflected waves arrive back at the hybrid with the same relative phasing. It is easy to show from the scattering parameters of an ideal hybrid network that this results in a cancellation of the reflected waves at the generator port, with the result that all of the reflected power is directed to the port connected to the dummy load (L).

Figure 3a) shows FDAC results comparing the magnitude $|\rho|$ of the ratio of reflected to forward voltage on the generator and antenna sides of the hybrid power splitter when the matching elements are set for a resistive loading $R^{\prime}=3.4 \Omega / m$ for each frequency shown and $R^{\prime}$ is then increased to $36.0 \Omega / \mathrm{m}$. At frequencies below $85 \mathrm{MHz},|\rho|$ is $<0.1$ at the generator, resulting in a reflected power of less than $1 \%$. Figure $3 \mathrm{~b}$ ) shows that more than half of the input power is absorbed in the dummy load. However, because of the short time duration of ELMs, $85 \%$ of the total power from the generator can still be coupled to the antenna, as determined from a time history of rf loading of a typical ELMing plasma having 10:1 variations in resistive loading.

An additional feature of this circuit is that it can be used to distinguish between arcs and ELMs. In figure 3, the reflection coefficient on both sides of a power splitter is again shown, this time at a fixed frequency of $60 \mathrm{MHz}$, when an arc having an impedance to ground of $1+10 \mathrm{j} \Omega$ is introduced at varying locations on one power splitter output line only. In this case there is not a canceling reflecting wave at the other output line and $|\rho|$ at the generator port is $\sim 1 / 2$ the value of $|\rho|$ at the output port connected to the arcing line.

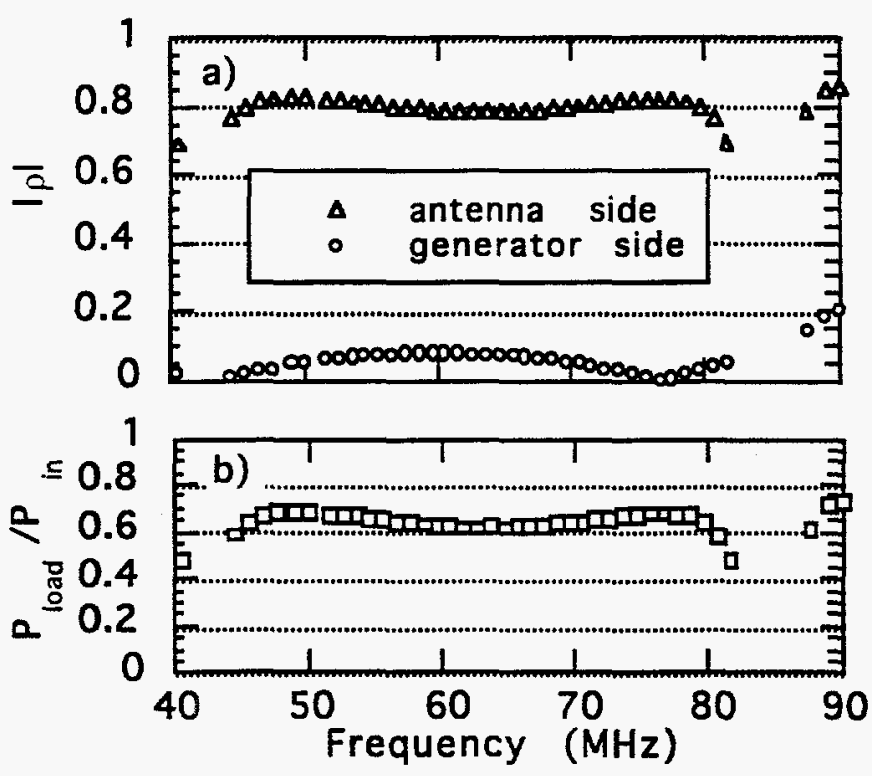

Figure 2. a) Power splitter reduces value of Irl produced by an ELM b) fraction of total input power diverted to dummy load during ELM. 


\section{THE TPX FEED CIRCUIT}

A diagram of the TPX feed circuit is shown in fig. 4. The design is based on the requirement of driving four antenna elements with each generator. This is accomplished by connecting top and bottom poloidal elements by a resonant loop for each of the 6 straps, with toroidal strap 1 , 2 , and 3 connected to toroidal straps 4,5 , and 6 respectively by a second tier of resonant loops. The line stretchers P1-P6 are used to adjust the length of the outer resonant loops for various operating frequencies, and keep the feed points at the impedance maxima. An FDAC calculation has shown that if the inner resonant loops are chosen each to be $3 \lambda$ long, and the outer loops to be $5 / 2 \lambda$ long at $f=60$ $\mathrm{MHz}$, then operating frequencies of $44.4,60$, and $75.6 \mathrm{MHz}$ can be obtained with fixed outer resonant loops. In the case of TPX, three decouplers (D1-D3) are required, as shown in the figure. With the outer resonant loops adjusted to give a phase difference of $\pi$ between pairs of connected straps, equal phase differences between

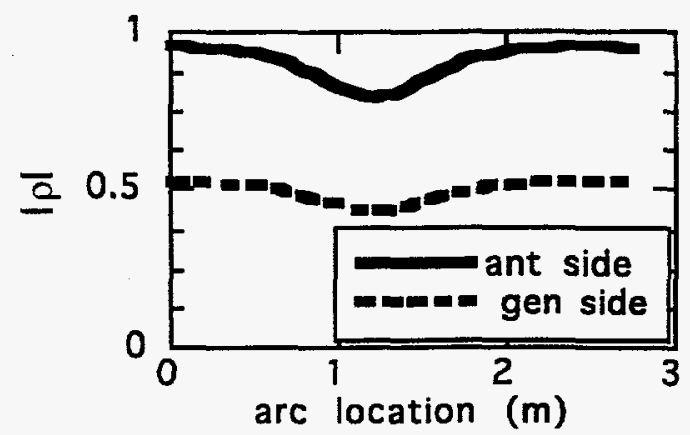

Fig. 3. Reflection coefficients on both sides of splitter with arc on one output leg.

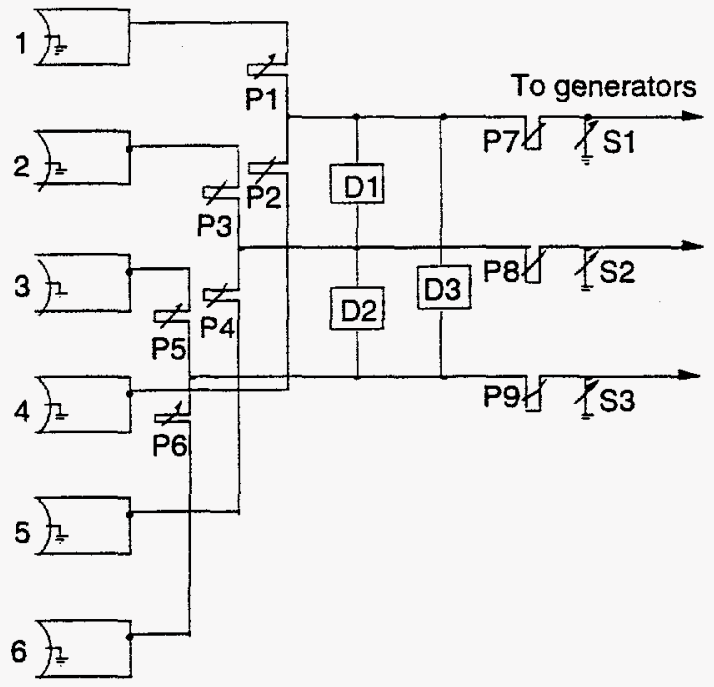

Fig. 4. TPX feed circuit Schematic neighboring straps can only be achieved for relative phases of $\pm \pi / 3^{\circ}$ and $\pm 2 \pi / 3$. The former phasing gives a highly directional spectrum having a toroidal mode number peak at $\mathrm{N}_{\mathrm{t}}=10{ }^{2}$ It is also possible to obtain symmetric spectra, with the best being produced by a strap phasing of $0,0,0, \pi, \pi, \pi$ which has a spectral peak at $\mathrm{N}_{\mathrm{t}}=14$. According to our model of the TPX system, the decoupler configuration shown allows an instantaneous change between these phases with no retuning, and without exceeding a VSWR value of 1.1 at the generators. Calculations using the RANT plasma loading code ${ }^{2}$ show that sufficient loading will be present at both phases to allow the full $6 \mathrm{MW}$ available power to be coupled to the antennas without exceeding an rf voltage of $26 \mathrm{kV}$ on any structure.

\section{REFERENCES}

1. "Ion Cyclotron Heating and Current Drive System", ITER Design Description Document DDD5.1 Section 1 for WBS 5.1, April 12, 1995.

2. "Milestone Report on the Ion Cyclotron System Tuning and Matching System", TPX report no. 23-950430-ORNL-DSwain-01 